\title{
MODELO MATEMÁTICO NO-UNEAL PARA UN SERVOSISTEMA NEUMÁTICO DE POSICIONAMIENTO PRECISO
}

\author{
Germán A. Bacca ${ }^{1}$ \\ VÍCTOR J. DE NEGRI ${ }^{2}$ \\ Yesid AssaF $^{3}$
}

\section{Resumen}

En este artículo se presenta en detalle el modelado matemático no-lineal de un servomecanismo neumático de posicionamiento. El servomecanismo neumático está compuesto en su estructura básica por un cilindro sin vástago gobernado por una válvula proporcional de vías. Para la elaboración del modelo se tuvo en cuenta la dinámica del cilindro, propiedades del fluido de trabajo y características de la válvula. El modelo de los flujos de masa a través de la válvula se basó en el modelo de la norma ISO 6358, y los parámetros se obtuvieron por medio de técnicas no-lineales de optimización a partir de datos experimentales tomados en estado transitorio. La validación del modelo dinámico del sistema se realizó para diferentes señales de entrada y se comparó con resultados obtenidos experimentalmente.

\section{Palabras clave}

Modelado matemático, servomecanismo neumático, fricción, área relativa, flujo de masa.

1 Ingeniero Mecánico, M.Sc., Profesor Asociado, Universidad del Cauca, Popayán, gbacca@unicauca.edu.co

2 Ingeniero Mecánico, Ph.D., Profesor Titular, Universidad Federal de Santa Catarina, Florianópolis-Brasil, victor@emc.ufsc.br

3 Ingeniero Mecánico, Ingeniero Investigador, Universidad Federal de Santa Catarina, Florianópolis-Brasil, yesid@emc.ufsc.br 


\section{Alostract}

In this paper, a nonlinear mathematical model of a servo pneumatic positioning system is developed in detail. The system is compound in its basic structure for a double-acting cylinder without piston rod which is controlled by a proportional valve. For the elaboration of model were taken in account the cylinder dynamics, work fluid properties and valve characteristics. The mass flow model is based on the model of standard ISO 6358, and the system parameters were estimated by means of nonlinear optimization techniques from experimental data in transient state. Validation of system dynamic model was made for different input signals and compared with experimental results.

\section{Keywords}

Mathematical model, pneumatic servomechanism, friction, relative area, mass flow. 


\section{INTRODUCCIÓN}

Los sistemas neumáticos han sido aplicados extensamente la automatización de maquinaria de producción y en el campo de los controladores automáticos. No obstante, factores como la compresibilidad del aire y la fricción, hacen que estos sistemas presenten una alta no-linealidad que dificulta indudablemente su estudio. En la actualidad, el estudio de los servomecanismos neumáticos tiene una gran relevancia debido a las grandes ventajas y aplicaciones que presentan estos sistemas. Sin embargo, el control de posición sobre todo en aplicaciones donde se requiera alta precisión, continúa siendo un reto. Las investigaciones realizadas en este campo están principalmente enfocadas hacia el modelado, la caracterización, y el análisis mediante técnicas computacionales de simulación de alguna componente disponible en el mercado (Nouri et al., 2000; Richer \& Hurmuzlu, 2000; Sorli et al., 2001; Ning \& Bone, 2005; Osama et al., 2005).

Igualmente, una gran parte de los estudios está enfocada en el modelado y el diseño de una estrategia de control a través de los cuales se procura lograr una elevada precisión tanto en el posicionamiento (Ning \& Bone, 2002; Bone \& Ning, 2005; Rao \& Bone, 2005), como en el control de fuerza del elemento actuador (Ilchman et al., 2000; Richer \& Hurmuzlu, 2000). En (Osama et al., 2005) se presenta la caracterización y el modelo analítico del flujo de masa en condiciones estáticas para una válvula proporcional de vías. La validación del modelo se realiza solamente en condiciones estáticas con buenos resultados. En (Sorli et al., 2001) se presenta el modelo dinámico de una válvula proporcional de presión. La validación del modelo queda pendiente para un futuro trabajo.

En (Ning \& Bone, 2005) se desarrolla un modelo paramétrico de un servomecanismo neumático, el cual, según la validación arroja buenos resultados, sin embargo, el modelo presenta mayor precisión bajo ciertas condiciones de operación que en otras. En (Nouri et al., 2000) se presenta el modelo dinámico y la identificación de un servomecanismo neumático con fricción. 
La simulación y la validación del modelo quedan pendientes para un trabajo futuro. De otra parte, el estudio de fenómenos no-lineales presentes en sistemas neumáticos, se ha realizado mediante investigaciones en las cuales por medio de un modelo matemático y técnicas experimentales se ha logrado detectar la presencia de fenómenos tales como adherencia-deslizamiento, oscilaciones autoexcitadas o ciclos límite y oscilaciones caóticas (Takahiro \& Manabu, 2000).

El objetivo de este artículo es el desarrollo de un modelo matemático no-lineal para un servomecanismo neumático de posicionamiento, el cual permita analizar su dinámica. Este trabajo se basa en los siguientes aspectos: Modelado matemático del sistema, identificación de parámetros del sistema y validación del modelo. En este artículo el modelado matemático del sistema se desarrolla en la sección 2. En la sección 3 se realiza la identificación de los parámetros del sistema. En la sección 4 se presenta la validación del modelo frente a datos experimentales, y en la sección 5 se encuentran las conclusiones. La nomenclatura y referencias bibliográficas aparecen al final del documento.

\section{Modelado matemático}

El servomecanismo neumático de posicionamiento se presenta en la Fig. 1. Este sistema está compuesto principalmente por un cilindro de doble efecto sin vástago tipo Festo DGPL-25-500-PPV-A como elemento de trabajo, este a su vez está controlado por una válvula proporcional de vías tipo Festo MPYE-5-1/8-010-B. Estos elementos se encuentran conectados por mangueras plásticas, además, el sistema cuenta con sensores de presión tipo Festo SDET22T-D10 a la salida de las cámaras del cilindro y un potenciómetro de desplazamiento lineal tipo Festo MLO-POT-500-TLF que sensa la posición del cilindro. 


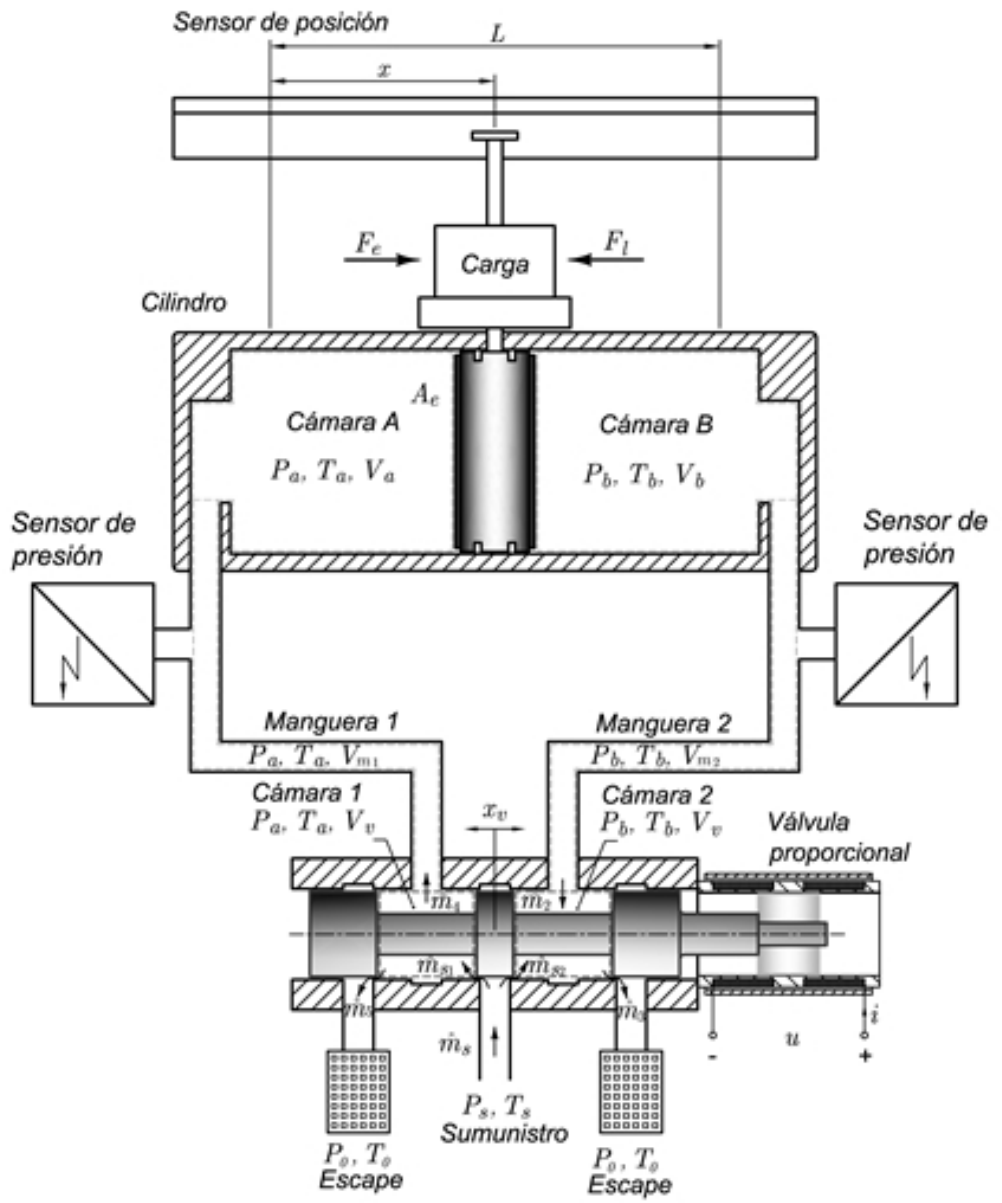

Fig. 1. Servomecanismo neumático de posicionamiento

Para el desarrollo del modelo matemático del sistema se han tendido en cuenta las siguientes suposiciones: El fluido de trabajo es aire y se considera como un gas ideal, se aplica la ley de conservación de la masa en cada volumen de control, se desprecian pérdidas de presión por fricción en los conductos de aire, y las propiedades como la densidad, la temperatura y la presión del aire se consideran uniformes en los diferentes volúmenes de control. 


\subsection{Modelado de la válvula proporcional de vías}

La válvula posee un diseño sencillo, robusto y económico, y algunas características de una servoválvula tales como buenas propiedades dinámicas y precisión. Por ser accionada directamente la corredera de la válvula, el fabricante denomina a esta válvula como válvula proporcional. Según su configuración física, esta válvula puede ser clasificada como sobretraslapada y tiene 3 posibles estados de flujo dependiendo de la posición que adopte su corredera. Puesto que la válvula tiene 5 conexiones (puertos), es una válvula 5/3 vías (Gerhartz \& Scholtz, 1995).

\subsection{Modelo de los flujos de masa}

En la Fig. 2 se muestra un diagrama en sección de la válvula en su posición media, en el cual se indican los sobretraslapes $x_{u i} \mathrm{y} x_{u o}$. A través del puerto 1 la válvula se comunica con la alimentación de aire comprimido. Los puertos 3 y 5 son de escape a la atmósfera. Por medio de los puertos de trabajo 2 y 4 la válvula se comunica a través de las mangueras con las cámaras del cilindro.

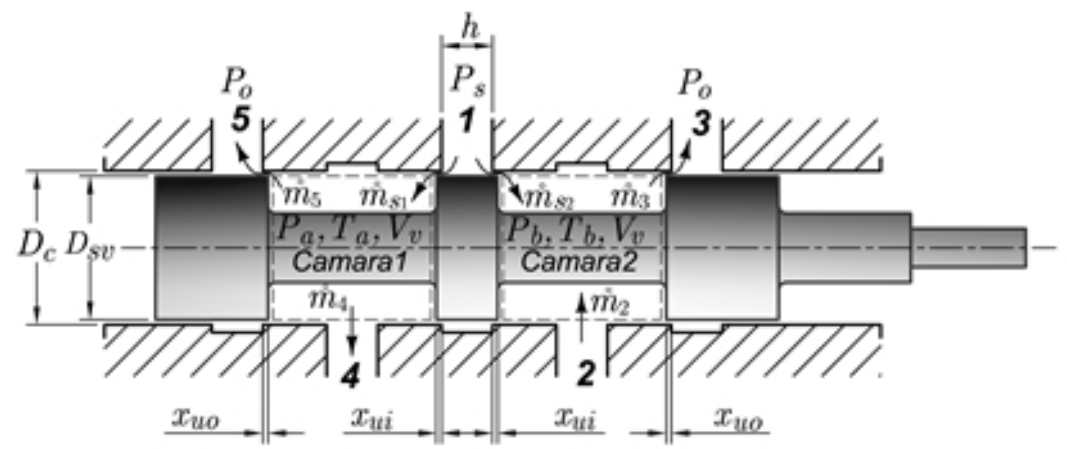

Fig. 2. Posición CENTRAL de LA VÁlvULA

Conectada la válvula al cilindro y bajo condiciones estables, los flujos de masa de suministro y en la posición media de la corredera son muy pequeños e iguales entre si, además, son prácticamente iguales a los flujos de masa de escape a la atmósfera 
$\stackrel{\circ}{m}_{5}$ y $\stackrel{\circ}{3}_{3}$, respectivamente, por tanto, los flujos de masa de trabajo $\stackrel{\circ}{m}_{4} \stackrel{\circ}{m}_{2}$ son iguales a cero. En esta posición la válvula se encuentra bajo condiciones de bloqueo. En la Fig. 3 se muestran los flujos de masa que se generan cuando la corredera tiene un desplazamiento $x_{v}^{+}$positivo. En este caso se produce un proceso de presurización en la cámara A del cilindro y un proceso de despresurización en la cámara B.

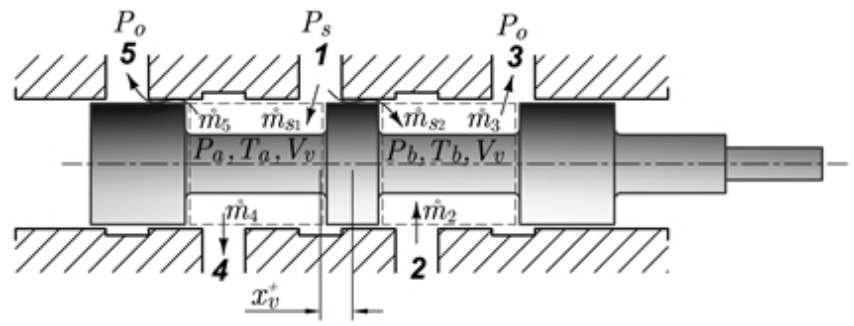

Fig. 3. Desplazamiento Positivo de LA CORREDeRA

En forma contraria a la anterior, cuando la corredera tiene un desplazamiento negativo, se generan los flujos de masa que se muestran en la Fig. 4. En esta situación se produce un proceso de despresurización en la cámara A y un proceso de presurización en la cámara B del cilindro.

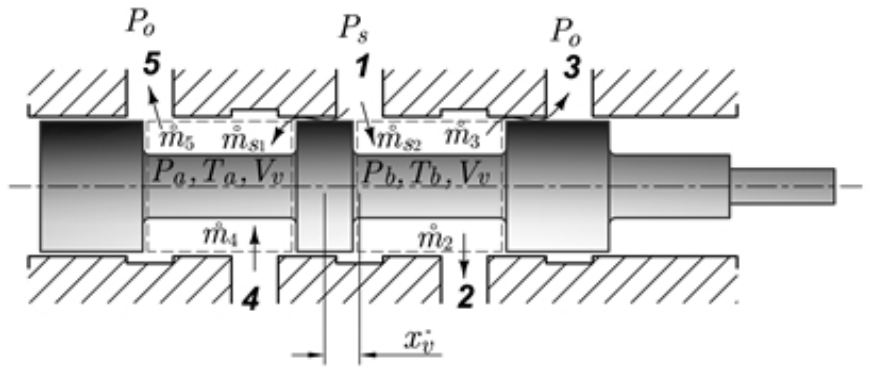

Fig. 4. Desplazamiento negativo de la corredera

Se puede establecer la analogía entre los cuatro orificios de área variable y las cuatro ramas de un puente de Wheatstone, tal como lo muestra la Fig. 5. Esta analogía permite obtener las ecuaciones de los flujos de masa. 


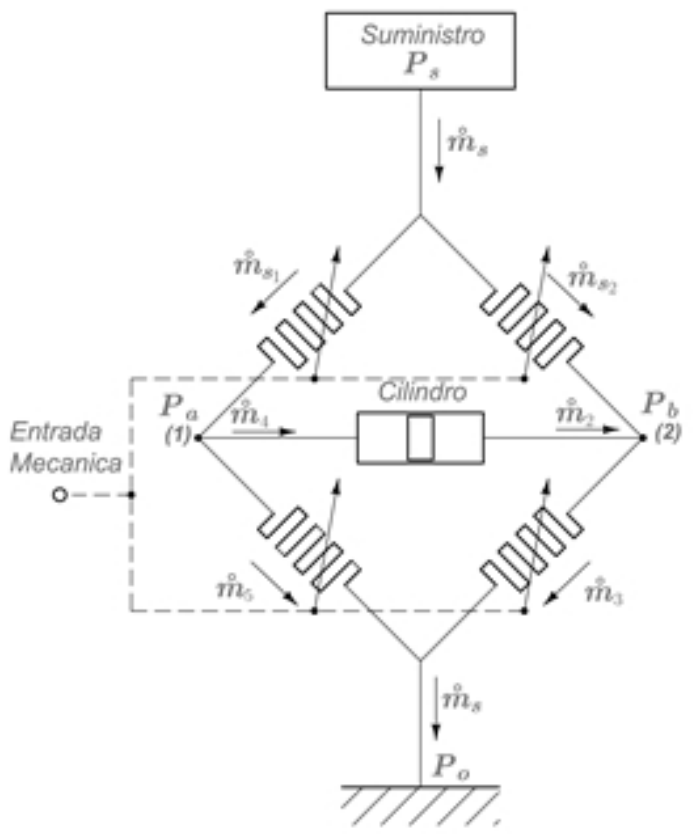

Fig. 5. Analogía electro-neumática de la válvula

Aplicando la ley de Kirchhoff a los flujos de masa que están presentes en los nodos (1) y (2) e igualando respectivamente a la rapidez de cambio de masa dentro de los volúmenes de control comprendidos en las cámaras 1 y 2 de la válvula, (véase la Fig. 2), se obtiene:

$$
\begin{aligned}
& {\stackrel{\circ}{m_{1}}}_{1}-\stackrel{\circ}{m}_{4}-\stackrel{\circ}{m}_{5}=\frac{V_{v}}{n R T_{a}} \frac{d P_{a}}{d t}=0 \\
& \stackrel{\circ}{m}_{s_{2}}+\stackrel{\circ}{m}_{2}-\stackrel{\circ}{m}_{3}=\frac{V_{v}}{n R T_{b}} \frac{d P_{b}}{d t}=0
\end{aligned}
$$

donde $P_{a}$ y $T_{a}$ son la presión y temperatura absolutas en el volumen de control A que comprende la cámara 1 de la válvula, la manguera 1 y la cámara A de cilindro, $P_{b}$ y $T_{b}$ son la presión y la temperatura absolutas en el volumen de control B que comprende la cámara 
2 de la válvula, la manguera 2 y la cámara $\mathrm{B}$ del cilindro, $v_{v}$ es el volumen en cada cámara del de la válvula (el cual es igual en cada cámara de la válvula y se despreciar), $R$ es la constante particular del aire y $\mathrm{n}$ es la constante politrópica del proceso.

El flujo de masa de aire a través de los puertos de la válvula se puede determinar por medio del modelo basado en la norma ISO 6358. Este modelo tiene dos parámetros que describen el flujo de masa: la relación de presiones crítica b, y la conductancia sónica C. El modelo se puede expresar como:

$$
\stackrel{\circ}{m}=A_{r} f\left(P_{u}, T_{u}, \frac{P_{d}}{P_{u}}\right)= \begin{cases}A_{r} f_{1}\left(P_{u}, T_{u}, \frac{P_{d}}{P_{u}}\right), & \frac{P_{d}}{P_{u}} \leq b \\ A_{r} f_{2}\left(P_{u}, T_{u}, \frac{P_{d}}{P_{u}}\right), & b<\frac{P_{d}}{P_{u}} \leq 0.999 \\ A_{r} f_{3}\left(P_{u}, T_{u}, \frac{P_{d}}{P_{u}}\right), & \frac{P_{d}}{P_{u}} \geq 0.999\end{cases}
$$

donde

$$
\begin{gathered}
f_{1}\left(P_{u}, T_{u}, \frac{P_{d}}{P_{u}}\right)=P_{u} C \rho_{n} \sqrt{\frac{T_{n}}{T_{u}}} \\
f_{2}\left(P_{u}, T_{u}, \frac{P_{d}}{P_{u}}\right)=P_{u} C \rho_{n} \sqrt{\frac{T_{n}}{T_{u}}} \sqrt{1-\left(\frac{\frac{P_{d}}{P_{u}}-b}{1-b}\right)^{2}} \\
f_{3}\left(P_{u}, T_{u}, \frac{P_{d}}{P_{u}}\right)=k_{1} P_{u}\left(1-\frac{P_{d}}{P_{u}}\right) \sqrt{\frac{T_{n}}{T_{u}}} \\
k_{1}=10^{3} C \rho_{n} \sqrt{1-\left(\frac{0.999-b}{1-b}\right)^{2}}
\end{gathered}
$$


$A_{r}$ es el área relativa de cada puerto, $P_{u}$ y $T_{u}$ son la presión y la temperatura absolutas en la sección de alta presión, $P_{d}$ es la presión absoluta en la sección de baja presión, $\rho_{n}$ y $T_{n}$ son la densidad y la temperatura del aire a las condiciones establecidas por la norma ISO $6358\left(\rho_{n}=1.18 \frac{\mathrm{kg}}{\mathrm{m}^{3}}\right.$ y $\left.T_{n}=293.15 \mathrm{~K}\right)$. Los estados de las variables en las secciones de alta y baja presión cambian de acuerdo a los procesos de presurización y de despresurización que experimenta cada cámara del cilindro en un determinado momento. Para el proceso de presurización en cualquier cámara, las condiciones de suministro son las de alta presión y las condiciones de la cámara son las de baja presión. En cambio para el proceso de despresurización, las condiciones de alta presión son las de la clamara y las de baja presión son las atmosféricas.

El área relativa se define como la relación entre el área geométrica variable del orificio de paso en un puerto de la válvula y el área geométrica máxima, o sea:

$$
A_{r}=\frac{A_{o}}{A_{o_{\max }}}
$$

Este parámetro es independiente de las presiones en el orificio, únicamente depende del voltaje aplicado a la válvula (Vieira, 1998). El cálculo de los flujos se realiza teniendo en cuenta la configuración mostrada en la Fig. 6.

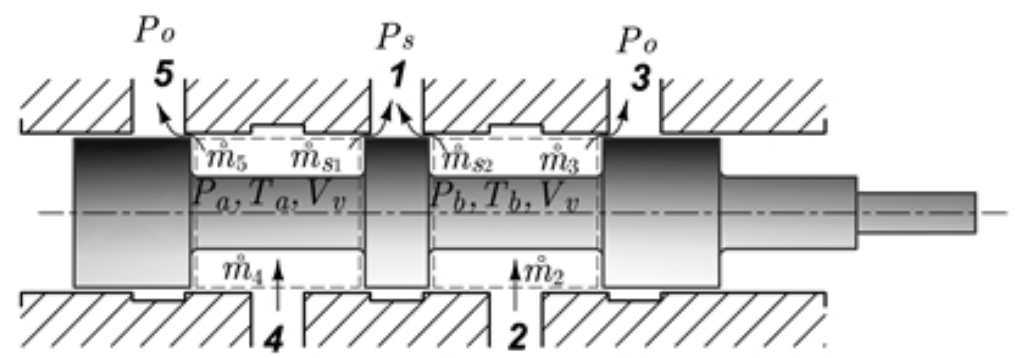

Fig. 6. Configuración de flujos de MASA PARA PUERTOS 4 y 2 
Según esta configuración, las ecuaciones de flujo de masa en estado estable en los puertos 4 y 2 se pueden escribir como:

$$
\begin{aligned}
& \stackrel{\circ}{m}_{4}=\stackrel{\circ}{m}_{s_{1}}+\stackrel{\circ}{m}_{5} \\
& \stackrel{\circ}{m}_{2}=\stackrel{\circ}{m}_{s_{2}}+\stackrel{\circ}{m}_{3}
\end{aligned}
$$

se definen las relaciones de flujo de masa:

$$
\begin{aligned}
& \alpha_{4}=\frac{{\stackrel{\circ}{s_{1}}}_{\dot{o}_{4}}}{\dot{m}_{4}} ; \beta_{4}=\frac{\stackrel{\circ}{m}_{5}}{\dot{\circ}_{4}} \text {, } \\
& \alpha_{2}=\frac{{\stackrel{\circ}{s_{2}}}_{\dot{\circ}_{2}}}{\dot{m}_{2}} ; \beta_{2}=\frac{\stackrel{\circ}{m}_{3}}{\dot{\circ}_{2}}
\end{aligned}
$$

por tanto:

$$
\begin{aligned}
& \alpha_{4}+\beta_{4}=1 \\
& \alpha_{2}+\beta_{2}=1
\end{aligned}
$$

Ahora, de (1) y (2) se tiene que:

$$
\begin{aligned}
& \stackrel{\circ}{m}_{4}={\stackrel{\circ}{m_{1}}}_{s_{1}}-\stackrel{\circ}{m}_{5}=A_{r s_{1}} f\left(P_{s}, T_{s}, \frac{P_{a}}{P_{s}}\right)-A_{r_{5}} f\left(P_{a}, T_{a}, \frac{P_{0}}{P_{a}}\right) \\
& \stackrel{\circ}{m}_{2}=\stackrel{\circ}{m}_{3}-\stackrel{\circ}{m}_{s_{2}}=A_{r_{3}} f\left(P_{b}, T_{b}, \frac{P_{0}}{P_{b}}\right)-A_{r s_{2}} f\left(P_{s}, T_{s}, \frac{P_{b}}{P_{s}}\right)
\end{aligned}
$$

se pueden establecer las siguientes relaciones:

$$
\begin{array}{ll}
\frac{{\stackrel{\circ}{s_{1}}}_{\dot{\circ}_{4}}}{\dot{m}_{4}}=\frac{A_{r s_{1}}}{A_{r_{4}}}=\alpha_{4} ; & \frac{\stackrel{\circ}{m}_{5}}{\stackrel{\circ}{m}_{4}}=\frac{A_{r_{5}}}{A_{r_{4}}}=\beta_{4} \\
\frac{\stackrel{\circ}{m}_{s_{2}}}{\stackrel{\circ}{m}_{2}}=\frac{A_{r s_{2}}}{A_{r_{2}}}=\alpha_{2} ; & \frac{\stackrel{\circ}{m}_{3}}{\stackrel{\circ}{m}_{2}}=\frac{A_{r_{3}}}{A_{r_{2}}}=\beta_{2}
\end{array}
$$

Por tanto, las ecuaciones de los flujos de masa a través de los puertos de trabajo de la válvula proporcional, se expresan como: 


$$
\begin{aligned}
& \stackrel{\circ}{m}_{4}=A_{r_{4}} \alpha_{4} f\left(P_{s}, T_{s}, \frac{P_{a}}{P_{s}}\right)-A_{r_{4}} \beta_{4} f\left(P_{a}, T_{a}, \frac{P_{0}}{P_{a}}\right) \\
& \stackrel{\circ}{m}_{2}=A_{r_{2}} \beta_{2} f\left(P_{b}, T_{b}, \frac{P_{0}}{P_{b}}\right)-A_{r_{2}} \alpha_{2} f\left(P_{s}, T_{s}, \frac{P_{b}}{P_{s}}\right)
\end{aligned}
$$

donde los parámetros $A_{r_{4}}, \alpha_{4}, \beta_{4}$ y $A_{r_{2}}, \alpha_{2}, \beta_{2}$ se determina experimentalmente. La válvula proporcional presenta un ancho de banda generalmente por encima de $100 \mathrm{~Hz}$, mientras que el ancho de banda en lazo cerrado del servo-neumático no supera los $10 \mathrm{~Hz}$, por tanto, la dinámica de la válvula se puede despreciar sin que esto afecte significativamente la precisión del modelo.

\subsection{Modelo del actuador}

\subsubsection{Modelo de los flujos de masa en las cámaras del actuador}

Aplicando la ecuación de continuidad a los volúmenes de control comprendidos en las cámaras A y B, se obtienen las ecuaciones:

$$
\begin{aligned}
& \stackrel{\circ}{m}_{a}=A_{e}\left[\frac{P_{a}}{R T_{a}} \frac{d x}{d t}+\frac{\left(x+x_{a r}\right)}{n R T_{a}} \frac{d P_{a}}{d t}\right] \\
& \stackrel{\circ}{m}_{b}=-A_{e}\left[-\frac{P_{b}}{R T_{b}} \frac{d x}{d t}+\frac{\left(L-x+x_{b r}\right)}{n R T_{b}} \frac{d P_{b}}{d t}\right]
\end{aligned}
$$

donde $\stackrel{\circ}{m}_{a}$ es el flujo de masa a través de la superficie de control de la cámara A, $\grave{m}_{b}$ es el flujo de masa a través de la superficie de control de la cámara $\mathrm{B}, A_{e}$ es el área del émbolo, $L$ es la carrera del cilindro, $x$ es el desplazamiento del émbolo, $x_{a r}=V_{a r} / A_{e}$ y $x_{b r}=V_{b r} / A_{e}$ son los desplazamientos remanentes equivalentes comprendidos entre el émbolo y la camisa del cilindro cuando $x=0$ y $x=L$, respectivamente. 


\subsubsection{Dinámica del émbolo}

En la Fig. 7 se muestran las fuerzas presentes en el émbolo, incluyendo las fuerzas de impacto en los finales de carrera del cilindro.

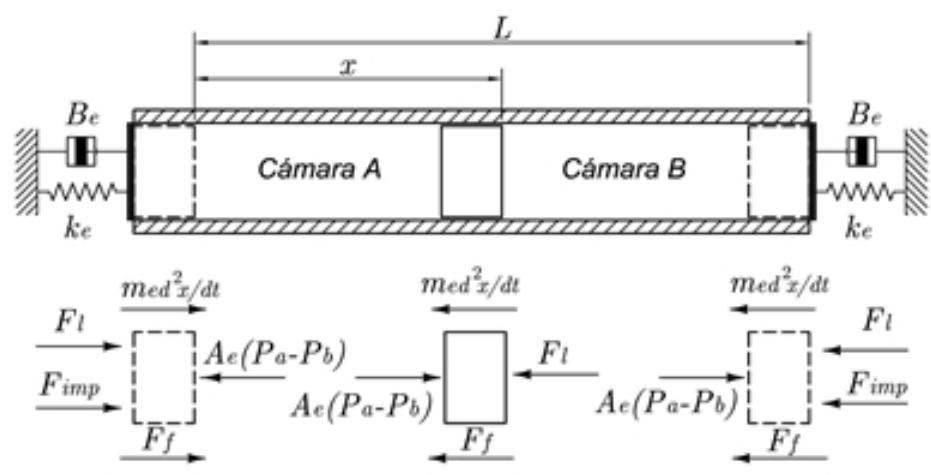

FIG. 7. DinÁMICA dEL ÉMBOLO

Aplicando la segunda ley de Newton, la dinámica del émbolo se puede expresar como:

$$
m_{e} \stackrel{\circ}{x}=\left\{\begin{array}{l}
A_{e}\left(P_{a}-P_{b}\right)-F_{f}-F_{l}-F_{i m p}, x<0 \text { o } x>L \\
A_{e}\left(P_{a}-P_{b}\right)-F_{f}-F_{l}, 0 \leq x \leq L
\end{array}\right.
$$

donde $A_{e}\left(P_{a}-P_{b}\right)$ es la fuerza efectiva en el émbolo debido a la diferencia de presiones en las cámaras A y B del cilindro, me es la masa del émbolo (incluido el carro del cilindro), $F_{l}$ es la fuerza de la carga externa, $F_{f}$ es la fuerza de fricción (seca + viscosa) y $F_{i m p}$ es la fuerza de impacto en los extremos. La fuerza de impacto se determina mediante el modelo basado en la teoría elastoplástica de los materiales:

$$
F_{i m p}=\left\{\begin{array}{l}
k_{e} x+B_{e} \stackrel{\circ}{x}, x<0 \\
k_{e}(x-l)+B_{e} \stackrel{\circ}{x}, x>L
\end{array}\right.
$$


donde $k_{e}$ es la rigidez equivalente y $B_{e}$ es el coeficiente de amortiguación equivalente. La fricción del sistema está representada por el modelo de LuGre (Lischinsky et al., 1999), el cual está definido como:

$$
\begin{aligned}
& \frac{d z}{d t}=v-\frac{\sigma_{0}|v| z}{F_{c}+\left(F_{s}-F_{c}\right) e^{-\left(\frac{v}{v_{s}}\right)^{2}}} \\
& F_{f}=\sigma_{0} z+\sigma_{1} \stackrel{\circ}{z}+B v
\end{aligned}
$$

donde $v=\stackrel{\circ}{x}$ es la velocidad del émbolo, $F_{s}$ es la fuerza de fricción estática, $F_{c}$ es la fuerza de fricción de Coulomb, $z$ es la deflexión media de cerda (bristle deflection), $v_{s}$ es la velocidad de deslizamiento, $\sigma_{0}$ es el coeficiente de rigidez, $\sigma_{l}$ es el coeficiente de amortiguamiento seco y $\mathrm{B}$ es el coeficiente de fricción viscosa.

\subsubsection{Modelo de los flujos de masa en las mangueras}

$\mathrm{Al}$ aplicar la ley de continuidad a los volúmenes de control comprendidos en las mangueras 1 y 2 (véase la Fig. 1) y asumiendo que el cambio en el volumen en las mangueras es despreciable, se obtienen las ecuaciones de flujo de masa:

$$
\begin{aligned}
& \stackrel{\circ}{m}_{4}-\stackrel{\circ}{m}_{a}=\frac{V_{m_{1}}}{n R T_{a}} \frac{d P_{a}}{d t} \\
& \stackrel{\circ}{m}_{b}-\stackrel{\circ}{m}_{2}=\frac{V_{m_{2}}}{n R T_{b}} \frac{d P_{b}}{d t}
\end{aligned}
$$

donde $v_{m_{1}}$ y $v_{m_{2}}$ son los volúmenes en las mangueras 1 y 2 , respectivamente.

\subsubsection{Dinámica de la presión en el sistema}

Teniendo en cuenta que la presión y la temperatura del aire son uniformes en los diferentes volúmenes de control (válvula- 
mangueras-cilindro), el modelo de la dinámica de la presión en todo el sistema se obtiene a partir de la combinación de las diferentes ecuaciones de flujo de masa obtenidas. Las ecuaciones de la dinámica de la presión del aire en los volúmenes de control de las clamaras A y B se expresan como:

$$
\begin{aligned}
& \frac{d P_{a}}{d t}=\frac{n}{x+x_{a r}+\frac{V_{m_{1}}}{A_{e}}}\left[\frac{R T_{a_{0}} P_{a}^{\frac{n-1}{n}}}{A_{e} P_{a_{0}}^{\frac{n-1}{n}}} \dot{\circ}_{4}+P_{a} v\right] \\
& \frac{d P_{b}}{d t}=\frac{n}{L-x+x_{b r}+\frac{V_{m_{2}}}{A_{e}}}\left[\frac{R T_{b_{0}} P_{b}^{\frac{n-1}{n}}}{A_{e} P_{b_{0}}^{\frac{n-1}{n}}} m_{2}+P_{b} v\right]
\end{aligned}
$$

donde $P_{a_{0}}$ y $T_{a_{0}}$ son los estados iniciales de la presión y temperatura en el volumen de control A, y, $P_{b_{0}}$ y $T_{b_{0}}$ son los estados iniciales de la presión y temperatura en el volumen de control B.

\section{IDENTIFICACIÓN DEL SISTEMA}

La identificación del sistema se centró principalmente en la caracterización de la válvula y en la obtención de los parámetros de fricción del actuador neumático por medio de pruebas experimentales.

\subsection{Parámetros de la válvula proporcional}

\subsection{1 Área relativa y relaciones de flujo de masa}

Estos parámetros se determinan experimentalmente mediante procesos de presurización y despresurización de una cámara de volumen conocido conectada a uno de los puertos de trabajo de la válvula. En cada prueba se registra la variación de la presión dentro de la cámara. La Fig. 8 muestra el esquema del montaje 
para el puerto 4, de igual manera, el montaje para el puerto 2 se realiza conectando el tanque de pruebas a este puerto y bloqueando la salida del puerto 4 .

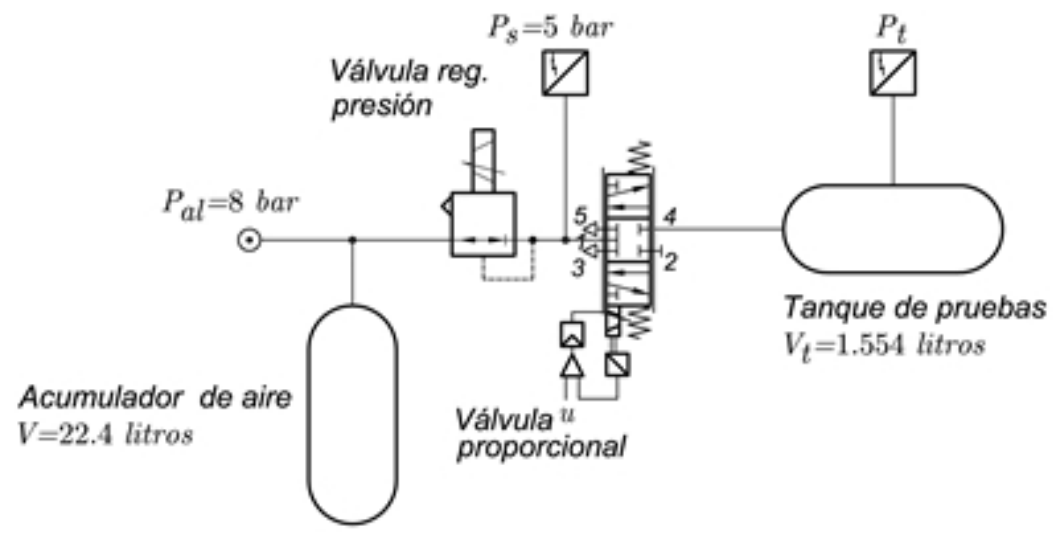

Fig. 8. Montaje de pruebas para el puerto 4

Para cada puerto de trabajo de la válvula se obtienen diferentes curvas de presurización y despresurización de acuerdo con los diferentes voltajes aplicados u. La presión inicial en la cámara se establece aplicando a la válvula un voltaje adecuado con el cual se puede igualar esta presión a la presión atmosférica para los procesos de presurización o a la presión de suministro para los procesos de despresurización. Es posible determinar los parámetros $A_{r_{4}}(u), \alpha_{4}(u), \beta_{4}(u)$ y $A_{r_{2}}(u), \alpha_{2}(u), \beta_{2}(u)$, mediante la comparación entre los datos experimentales de la variación de la presión en la cámara y los datos obtenidos por simulación numérica a partir de las ecuaciones:

$$
\begin{aligned}
& \frac{d P_{t}}{d t}=\frac{n R T_{t}}{V_{t}}\left[A_{r_{4}}(u) \alpha_{4}(u) f\left(P_{s}, T_{s}, \frac{P_{t}}{P_{s}}\right)-A_{r_{4}}(u) \beta_{4}(u) f\left(P_{t}, T_{t}, \frac{P_{0}}{P_{t}}\right)\right] \\
& \frac{d P_{t}}{d t}=\frac{n R T_{t}}{V_{t}}\left[A_{r_{2}}(u) \alpha_{2}(u) f\left(P_{s}, T_{s}, \frac{P_{t}}{P_{s}}\right)-A_{r_{2}}(u) \beta_{2}(u) f\left(P_{t}, T_{t}, \frac{P_{0}}{P_{t}}\right)\right]
\end{aligned}
$$


donde $V_{t}$ es el volumen de la cámara, $P_{t}$ es la presión dentro de la cámara y $T_{t}$ es la temperatura dentro de la cámara, la cual es constante ya que se consideran los procesos de presurización y despresurización isotérmicos $(n=1)$. Para la simulación de (27) y (28) se tienen en cuenta las condiciones en las que se realizaron las pruebas experimentales, estas son: $P_{s}=5.797 \mathrm{bar}, T_{s}=295 \mathrm{~K}$, $P_{0}=0.797 \mathrm{bar}, T_{0}=295 \mathrm{Ky} V_{t}=1.554 \mathrm{lit}$.

La Fig. 9 muestra una curva característica de la variación de la presión en la cámara conectada al puerto de trabajo 4 en un proceso de presurización y su correspondiente curva teórica obtenida por simulación numérica de (27), donde los parámetros $A_{r_{4 p}}, \alpha_{4 p}$ y $\beta_{4 p}$ se obtuvieron mediante el método de optimización no-lineal de minimos cuadrados.

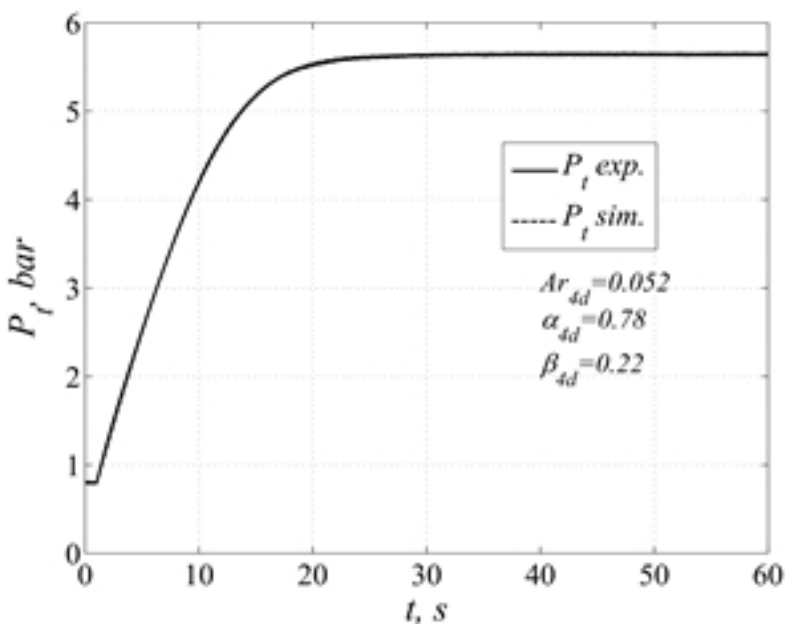

Fig. 9. CuRVA de PRESURIZACIÓN EN LA CÁmARA DE PRUEBAS, u $=5.5 \mathrm{~V}$

De las curvas experimentales de la variación de la presión en la cámara se comprueba que el área relativa está relacionada con el comportamiento transitorio y las relaciones de flujo de masa con el comportamiento en estado estacionario. Aplicando el método de optimización a las curvas $P_{t} v s$. $t$ en el rango del voltaje de entrada $u,[0,10] V$ fueron obtenidas las curvas de área relativa 
y de relaciones de flujo de masa para cada voltaje aplicado (véase la Fig. 10). Estas curvas fueron determinadas para los puertos de trabajo 4 y 2 , respectivamente, tanto para presurización como para despresurización del tanque de pruebas.

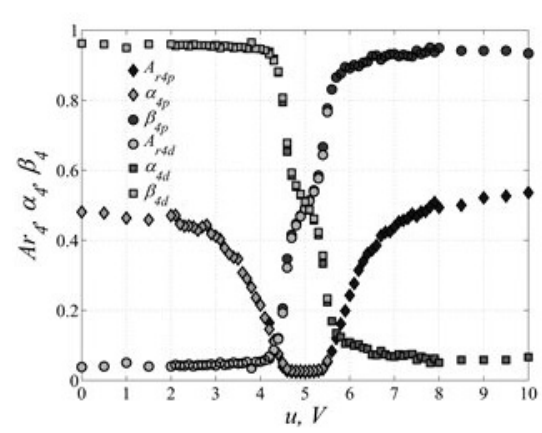

a)

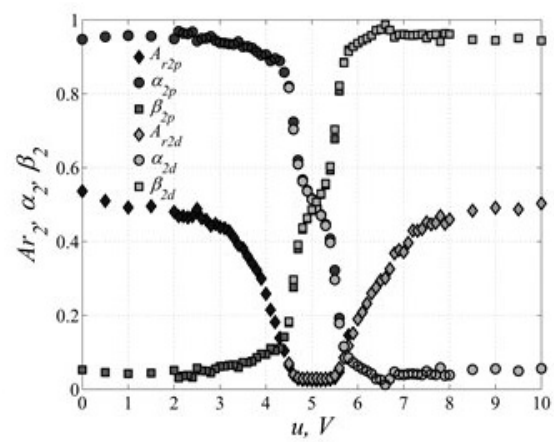

b)

Fig. 10. Área relativa y relaciones de flujo, a) Puerto 4 y b) Puerto 2

\subsubsection{Parámetros del cilindro}

Los parámetros de fricción del cilindro se estimaron de acuerdo con el modelo de LuGre, el cual en estado estacionario se expresa como:

$$
F_{s s_{i}}=\left[F_{c}+\left(F_{s}-F_{c}\right) e^{-\left(\frac{v_{s s_{i}}}{v_{s}}\right)^{2}}\right] \operatorname{sign}\left(v_{s s_{i}}\right)+B v_{s s_{i}}
$$

En la Fig. 11 se muestra el mapa de fuerza vs. velocidad en estado estacionario, tanto para velocidades positivas como para negativas. Los parámetros $F_{s}, F_{c}, B$ y $v_{s}$ se estimaron por medio de la técnica de optimización no-lineal de mínimos cuadrados. 


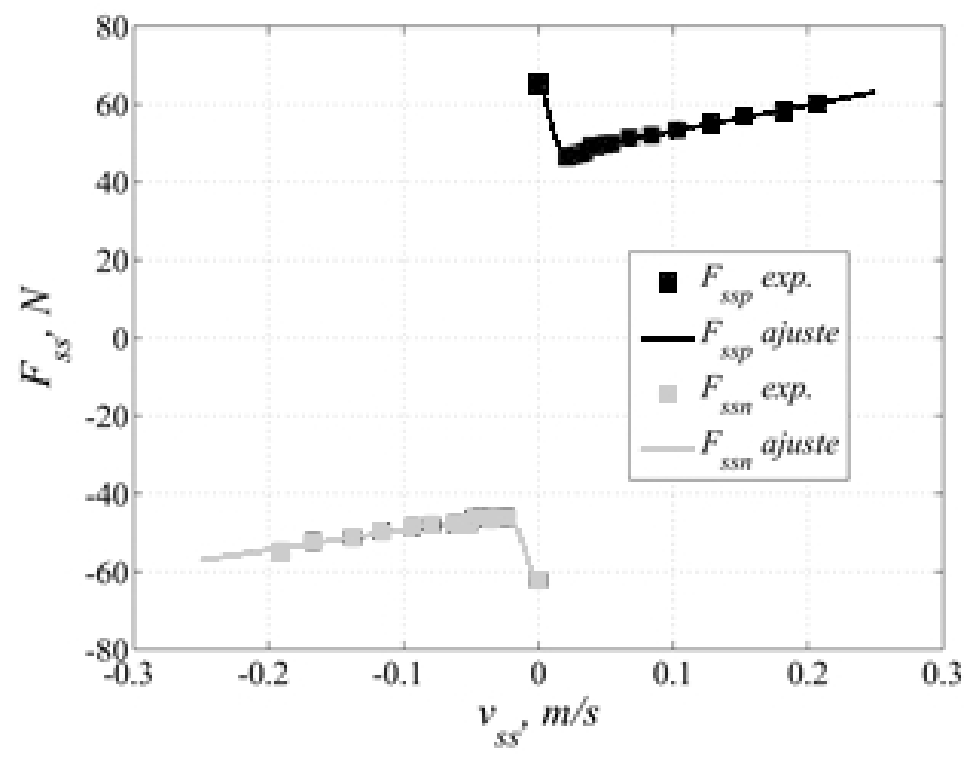

Fig. 11. MAPA DE FRICCIÓn En ESTAdo EStAcIONARIO

Los parámetros estimados son: $F_{s p}=65.4 \mathrm{~N}, F_{c p}=745.7 \mathrm{~N}$, $B_{p}=72 \mathrm{~N} . \mathrm{s} / \mathrm{m}, v_{s_{p}}=0.011 \mathrm{~m} / \mathrm{s}, F_{s_{n}}=-62.2 \mathrm{~N}, F_{c_{n}}=-44.3 \mathrm{~N}, B_{n}=50.8$ $N . s / m, v_{s_{p}}=0.013 \mathrm{~m} / \mathrm{s}$, donde los subíndices $p$ y $n$ indican velocidades positivas y negativas, respectivamente. La estimación de los parámetros $\sigma_{0}$ y $\sigma_{I}$ no es posible mediante técnicas lineales debido a la dependencia no-lineal de la fricción con estos dos parámetros, $\mathrm{y}$ al hecho que el estado interno $z_{i}$ no es medible (Lischinsky et al., 1999).

El parámetro $\sigma_{0}$ se puede estimar directamente a partir de la relación:

$$
\sigma_{0_{i}}=\frac{\Delta F_{i}}{\Delta x_{i}}
$$

$\triangle F_{i}$ es la fuerza diferencial necesaria para producir microdesplazamientos diferenciales pre-deslizamiento $\triangle x_{1}$ en el émbolo. Debido a que no se cuenta con los instrumentos de medida adecuados, 
es imposible medir estos microdesplazamientos. Por tanto, el parámetro $\sigma_{0}$ se calcula de forma aproximada asumiendo que el émbolo presenta microdesplazamientos con movimiento de predeslizamiento cuando se aplica una fuerza $F$ menor que la fuerza de rompimiento a la que el émbolo empieza a deslizarse. Se asume entonces que $\stackrel{\circ}{x} \approx 0, \stackrel{\circ}{x} \approx 0$, y $\stackrel{\circ}{z}$ constante. De (22):

$$
F \approx \sigma_{0} z
$$

Así, reemplazando (31) en (21), se obtiene:

$$
\frac{d z}{d t}=\stackrel{\circ}{x}-\frac{F|\grave{x}|}{F_{s}}
$$

Esta ecuación se puede integrar en el intervalo $(0, \mathrm{~T})$ con $F(t)=\frac{0.92 F_{s}}{T} t, \quad \stackrel{\circ}{x}(t)=\frac{x_{\lim }}{T} t, \stackrel{\circ}{x}_{\text {lim }}$, es la menor velocidad obtenida del mapa de fricción en estado estacionario, y la condición inicial. Simulando (32) en el intervalo $(0,0.1) s$, se puede estimar el valor de $\sigma_{0}$ ajustando los resultados a la relación lineal $F(t)=\sigma_{0} z(t)$. El coeficiente $\sigma_{l}$ se estima considerando que el sistema tiene un amortiguamiento cercano al crítico $(0.8 \leq \xi \leq 1)$, mediante la expresión: $\sigma_{1}=2 \xi \sqrt{\sigma_{0} m_{e}}-B$.

Aplicando el anterior procedimiento con $\xi=0.8$ se obtienen los parámetros: $\sigma_{0_{p}}=1.27 \times 10^{6} \mathrm{~N} / \mathrm{m}, \sigma_{1_{p}}=1127.9 \mathrm{~N} . \mathrm{s} / \mathrm{m}$, $\sigma_{0_{n}}=1.29 \times 10^{6} \mathrm{~N} / \mathrm{m}, \sigma_{1_{n}}=1054.6 \mathrm{~N} . \mathrm{s} / \mathrm{m}$.

\section{VALIDACIÓN DEL MODELO}

En la validación del modelo se compara los datos experimentales con los resultados simulados para entradas paso, rampa y seno. Es de importancia anotar que en las simulaciones se considera que los procesos termodinámicos en los volúmenes de control son isotérmicos con temperaturas iniciales: $T_{a_{0}}=T_{b_{0}}=T_{o}=295 \mathrm{~K}$. La Fig. 12 muestra la respuesta experimental y la teórica par una entrada paso, donde 
$u(t)= \begin{cases}5.0 \mathrm{~V}, & 0 \leq t<1 \\ 5.5 \mathrm{~V}, & t \geq 1\end{cases}$

y condiciones iniciales: $P_{a_{0}}=4.26$ bar, $P_{b_{0}}=4.41 .4$ bar $y x_{0}=0.1094 \mathrm{~m}$.
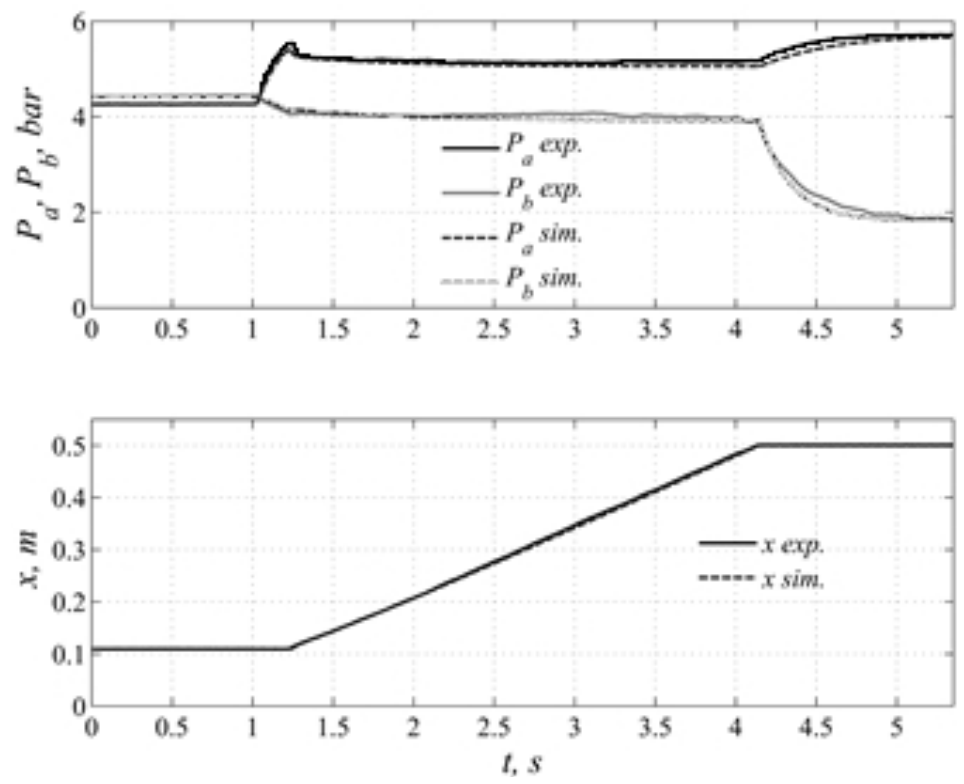

FIG. 12. RESPUESTA PASO, $U=5.5 \mathrm{~V}$

La respuesta pasó con

$$
u(t)=\left\{\begin{array}{lc}
4.0 \mathrm{~V}, & 0 \leq t<1 \\
5.56 \mathrm{~V}, & t \geq 1
\end{array}\right.
$$

y condiciones iniciales: $P_{a_{0}}=0.797$ bar, $P_{b_{0}}=5.797$ bar $y x_{0}=0 \mathrm{~m}$, se muestra en la Fig. 13. 

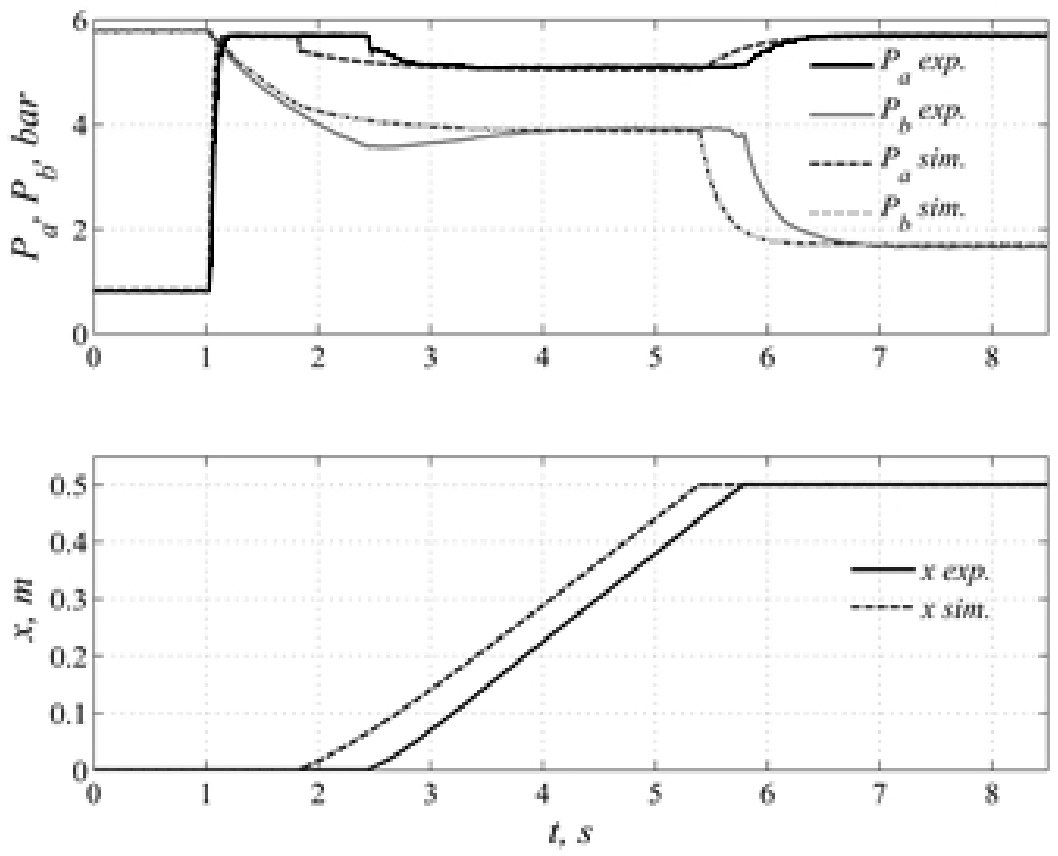

Fig. 13. Respuesta PAso, u $=5.56 \mathrm{~V}$

La respuesta a la entrada rampa se muestra en la Fig. 14 donde

$$
u(t)= \begin{cases}5.0 V, & 0 \leq t<1 \\ m_{i} t V, & t \geq 1\end{cases}
$$

$m_{i}=1 \mathrm{~V} / \mathrm{s}$ y condiciones iniciales: $P_{a_{0}}=4.228 \mathrm{bar}, P_{b_{0}}=4.441$ bar $y$ $x_{0}=0 \mathrm{~m}$.

Finalmente, la respuesta seno se muestra en la Fig. 15 donde

$$
u(t)=\left\{\begin{array}{l}
5.0 V, \quad 0 \leq t<1 \\
5.0+A_{i} \operatorname{sen}\left(2 \pi f_{i} t\right) V, \quad t \geq 1
\end{array}\right.
$$


$A i=0.8 \mathrm{~V}, f_{i}=1 \mathrm{~Hz}$ y condiciones iniciales: $P_{a}=4.213 \mathrm{bar}, P_{b_{0}}=$ 4.328 bar y $x_{0}=0.25 \mathrm{~m}$.
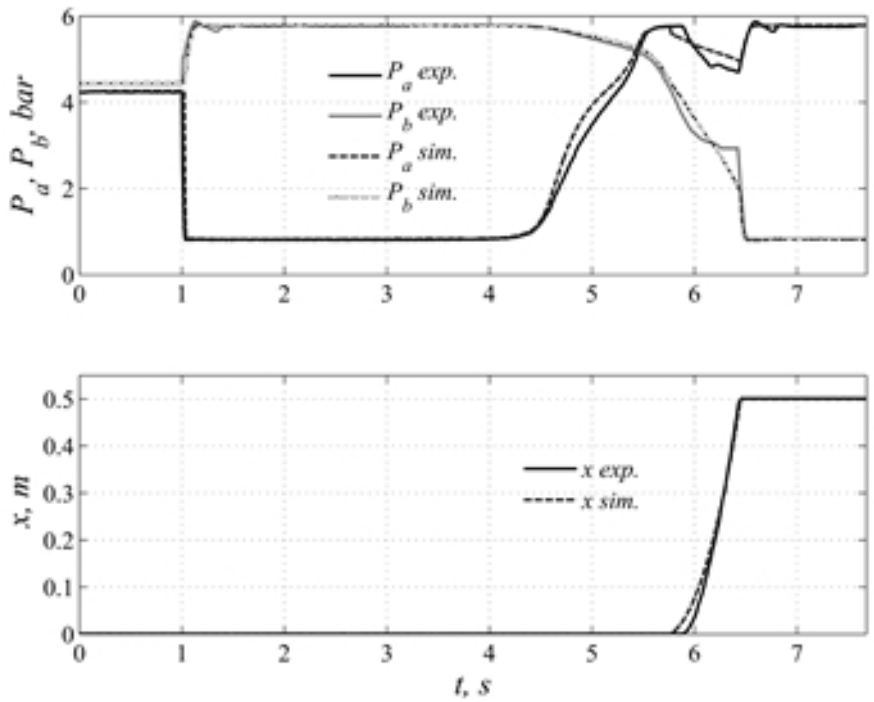

Fig. 14. Respuesta Rampa, U $=\mathrm{T} V$
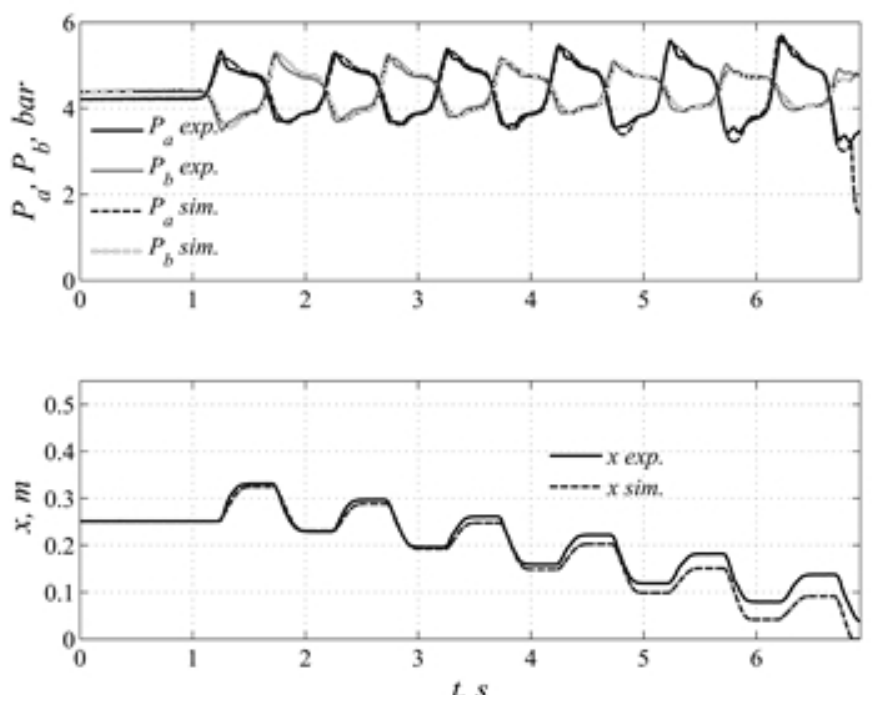

Fig. 15. Respuesta Seno, u $=5.0+0.8 \operatorname{sen}(2 \pi t)$ 
En general, de las respuestas del sistema frente a las diferentes entradas se observa que los resultados teóricos presentan una buena correspondencia con respecto a los datos experimentales. En la Fig. 13 es evidente la presencia de un adelanto en el tiempo de los resultados teóricos frente a los datos experimentales, esto se debe principalmente a que el comportamiento de la fricción en el émbolo no es uniforme a lo largo de su recorrido, siendo esta mayor cerca de uno de los finales de carrera del cilindro. Este fenómeno también se manifiesta en la respuesta seno haciendo que los resultados teóricos difieran cada vez mías de los datos experimentales a medida que transcurre el tiempo.

\section{COnCLUSiOnes}

En este artículo se ha presentado etapa por etapa el desarrollo de un modelo matemático no-lineal de un servomecanismo neumático de posicionamiento. Este modelo permitirá más adelante realizar el análisis de la dinámica no-lineal del sistema. Los parámetros del modelo se estimaron sin tener que recurrir a equipos de medida sofisticados ni a hardware especializado.

Además, en la caracterización de la válvula se comprobó que las pruebas en estado transitorio se pueden realizar con mayor facilidad y tienen mejor repetibilidad que las pruebas en estado estacionario. Las simulaciones demostraron que el modelo dinámico del servomecanismo presenta una buena correspondencia con los resultados experimentales. Con esto se demuestra que tanto el modelo de flujo de masa de la norma ISO 6358, como el modelo de fricción de LuGre son los más precisos. Además, se comprobó que la imprecisión del modelo se debe principalmente a la incertidumbre paramétrica entre los parámetros reales de fricción que varían con el recorrido del émbolo y los parámetros de fricción estimados, que son valores promedio y además uniformes.

En general, los resultados obtenidos en las simulaciones del modelo dinámico son satisfactorios, lo cual implica que el modelo puede ser utilizado para el posterior análisis de la dinámica no-lineal y el control del servosistema. 


\section{Nomenclatura}

$P_{s}, T_{s}:$ Condiciones de suministro, bar, $\mathrm{K}$.

$P_{0}, T_{0}$ : Condiciones atmosféricas, bar, K. $P_{a}, P_{b}$ : Presiones absolutas dentro de los volúmenes de control A y B, respectivamente, bar.

$T_{a}, T_{b}$ : Temperaturas absolutas dentro de los volúmenes de control A y B, respectivamente, $\mathrm{K}$.

$P_{a_{0}}, P_{b_{0}}$ : Presiones absolutas iniciales dentro de los volúmenes de control A y $\mathrm{B}$, respectivamente, $\mathrm{K}$.

$T_{a_{0}}, T_{b_{0}}$ : Temperaturas absolutas iniciales dentro de los volúmenes de control A y B, respectivamente, $\mathrm{K}$.

$x$ : Desplazamiento del émbolo, $\mathrm{m}$.

$v$ : Desplazamiento del émbolo, $\mathrm{m} / \mathrm{s}$.

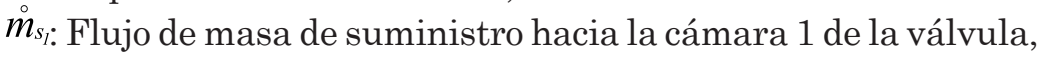
$\mathrm{kg} / \mathrm{s}$.

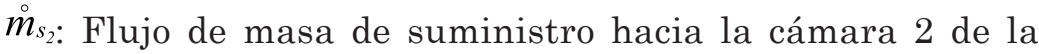
válvula, $\mathrm{kg} / \mathrm{s}$.

$\stackrel{\circ}{m}_{a}$ : Flujo de masa a través de la superficie de control de la cámara A del cilindro, $\mathrm{kg} / \mathrm{s}$.

$\stackrel{\circ}{m}_{b}$ : Flujo de masa a través de la superficie de control de la cámara $\mathrm{B}$ del cilindro, $\mathrm{kg} / \mathrm{s}$.

$\stackrel{\circ}{m}_{2}$ : Flujo de masa a través del puerto de trabajo 2 de la válvula, $\mathrm{kg} / \mathrm{s}$.

$\stackrel{\circ}{m}_{4}$ Flujo de masa a través del puerto de trabajo 4 de la válvula, $\mathrm{kg} / \mathrm{s}$.

$A_{r}:$ Área relativa, adimensional.

$\alpha, \beta$ : Relaciones de flujo de masa, adimensionales.

$n$ : Constante politrópica, adimensional.

$A_{e}$ : Área efectiva del émbolo, $\mathrm{m}^{2}$.

$x_{a r}, x_{b r}$ : Desplazamientos remanentes en las cámaras A y B del cilindro, respectivamente, $\mathrm{m}$.

$V_{m_{l}}, V_{m_{2}}$ : Volúmenes de las mangueras 1 y 2 , respectivamente, $\mathrm{m}^{3}$.

$V_{v}:$ Volumen en cada cámara de la válvula, $\mathrm{m}^{3}$. 
L: Carrera del cilindro, $\mathrm{m}$.

$m_{e}:$ Masa del émbolo, $\mathrm{kg}$.

$F_{f}$ Fuerza de fricción, $\mathrm{N}$.

$z$ : Deflexión media de cerda, $\mathrm{m}$.

$F_{s}$ : Fuerza de fricción estática, N.

$B$ : Coeficiente de fricción viscosa, $\mathrm{Ns} / \mathrm{m}$.

$\sigma_{0}$ : Coeficiente de rigidez, $\mathrm{N} / \mathrm{m}$.

$\sigma_{1}$ : Coeficiente de fricción seca, $\mathrm{Ns} / \mathrm{m}$.

$v_{s}$ : Velocidad de Stribeck, $\mathrm{m} / \mathrm{s}$.

$P_{u}$ : Presión de alta, bar.

$P_{d}$ : Presión de baja, bar.

$C$ : Conductancia sónica, $\mathrm{m}^{3} / \mathrm{s}$.bar.

$b$ : Relación de presiones crítica, adimensional.

$x_{u}$ : Sobretraslape, $\mathrm{m}$.

$u$ : Voltaje aplicado a la válvula, V.

\section{ReFERENCIAS}

Bone, G.M., Ning, S., (2005); Experimental Comparison of two Pneumatic Servo Positioning Control Algotithm, IEEE International Conference on Mechatronics and Automation, 1, 37-42.

Gerhartz, J., Scholtz, D., (1995); Neumática en Bucle Cerrado, Festo Didactic KG. D-73734 Esslingen 1.

Ilchman, A., Sawodny, O., Trenn, S., (2006); Pneumatic Cilinders: Modelling and Feedback Force-Control, International Journal of Control, 79(6), 650-661.

Lischinsky, P., De Wit, C.C., Morel, G., (1999); Friction Compensation for an Industrial Hydraulic Robot, IEEE Control Systems, 25-32.

Ning, S., Bone, G.M., (2002); High Steady-State Accuracy Pneumatic Servo Positioning System with PVA/PV Control and Friction Compensation, IEEE International Conference on Mechatronics and Automation, 3, 2824-2829.

Ning, S., Bone, G.M., (2005); Development of Nonlinear Dynamic Model for a Servo Pneumatic Positioning System, IEEE International Conference on Mechatronics and Automation, 1, 43-48. 
Nouri, B., Al-Bender, F., Swevers, J., Vanherck, P., Vanbrussei, H., (2000); Modeling a Pneumatic Servo Positioning System With Friction, American Control Conference, 1067-1071.

Osama, O., Brun, X., Sesmat, S., Redarce, T., Bideaux, E., (2005); Characterization And Modeling of a Proportional Valve for Control Synthesis, $6^{\text {th }}$ JFPS International Symposium on Fluid Power, Tsukuba, 771-776.

Rao, Z., Bone, G.M., (2005); Modeling and control of a miniature servo pneumatic actuator, IEEE International Conference on Robotics and Automation, 1, 37-42.

Richer, E., Hurmuzlu, Y., (2000); A High Performance Pneumatic Force Actuator System Part 1 - Nonlinear Mathematical Mode, ASME Journal of Dynamics Systems Measurement and Control, 122(3), 416-425.

Richer, E., Hurmuzlu, Y., (2000); A High Performance Pneumatic Force Actuator System Part 2 - Nonlinear Controller Design, ASME Journal of Dynamics Systems Measurement and Control, 122(3), 426-434.

Sorli, M., Figliolini, G., Pastorelly, S., (2001); Dynamic Model of a Pneumatic Proportional Pressure Valve, 2001 IEEE/ASME International Conference of advanced Intelligent Mechatronics Proceedings, 630-635.

Takashiro, K., Manabu, S., (2000); An Analytical and Experimental Study of Chaotic Oscillation in a Pneumatic Cylinder, 1st FPNI-PhD Symp., Hamburg, 303- 310.

Vieira, A.D., (1998); Análise Teórico Experimental de Servoposicionadores Lineares Pneumaticos, Master's thesis, Universisdade Federal de Santa Catarina, Curso De Pós-Gradução Em Engenharia Mecânica. 